\title{
Expression of the oxygen-regulated protein ORP150 accelerates wound healing by modulating intracellular VEGF transport
}

\author{
Kentaro Ozawa, ${ }^{1,2}$ Toshikazu Kondo, ${ }^{3}$ Osamu Hori, ${ }^{1,2}$ Yasuko Kitao, ${ }^{1}$ David M. Stern, ${ }^{4}$ \\ Wolfgang Eisenmenger, ${ }^{5}$ Satoshi Ogawa, ${ }^{1,2}$ and Tohru Ohshima ${ }^{3}$

\begin{abstract}
${ }^{1}$ Department of Neuroanatomy, Faculty of Medicine, Kanazawa University, Kanazawa City, Ishikawa, Japan ${ }^{2}$ Core Research for Engineering, Science, and Technology (CREST), Japan Science and Technology, Kawaguchi, Japan ${ }^{3}$ Department of Legal Medicine, Faculty of Medicine, Kanazawa University, Kanazawa City, Ishikawa, Japan

${ }^{4}$ Department of Physiology and Cellular Biophysics, College of Physicians and Surgeons, Columbia University, New York, New York, USA
\end{abstract} \\ ${ }^{5}$ Department of Legal Medicine, University of Munich, Munich, Germany
}

Address correspondence to: Kentaro Ozawa, Department of Neuroanatomy, Kanazawa University Medical School, 13-1 Takara-machi Kanazawa City, 920-8640 Ishikawa, Japan.

Phone: 81-76-265-2162; Fax: 81-76-234-4222; E-mail: ozawa@nanat.m.kanazawa-u.ac.jp.

Kentaro Ozawa and Toshikazu Kondo contributed equally to this work.

Received for publication November 13, 2000, and accepted in revised form May 14, 2001.

Expression of angiogenic factors such as VEGF under conditions of hypoxia or other kinds of cell stress contributes to neovascularization during wound healing. The inducible endoplasmic reticulum chaperone oxygen-regulated protein 150 (ORP150) is expressed in human wounds along with VEGF. Colocalization of these two molecules was observed in macrophages in the neovasculature, suggesting a role of ORP150 in the promotion of angiogenesis. Local administration of ORP150 sense adenovirus to wounds of diabetic mice, a treatment that efficiently targeted this gene product to the macrophages of wound beds, increased VEGF antigen in wounds and accelerated repair and neovascularization. In cultured human macrophages, inhibition of ORP150 expression caused retention of VEGF antigen within the endoplasmic reticulum (ER), while overexpression of ORP150 promoted the secretion of VEGF into hypoxic culture supernatants. Taken together, these data suggest an important role for ORP150 in the setting of impaired wound repair and identify a key, inducible chaperone-like molecule in the ER. This novel facet of the angiogenic response may be amenable to therapeutic manipulation.

J. Clin. Invest. 108:41-50 (2001). DOI:10.1172/JCI200111772.

\section{Introduction}

Angiogenesis occurs during development and represents a physiological response to environmental cues (1). Neovessel formation also occurs in response to stress (wound repair) (2) and in pathological situations later in life, such as tumor formation $(3,4)$ and recanalization of thrombi after ischemic events (5). Among the factors that mediate angiogenesis, VEGF has been the subject of extensive research because of its selective effect on endothelial cells (6). As a part of the adaptive responses that achieve angiogenesis, hypoxia-mediated induction of VEGF has been demonstrated in a wide variety of cells, including tumor cells (7), astrocytes (8), and cardiomyocytes (9). Furthermore, expression of receptors for angiogenic factors is also enhanced under hypoxia (10).

Exposure of cells to hypoxia triggers another series of responses, including induction of a set of polypeptides termed oxygen-regulated proteins (ORPs) (11). In this context, astrocytes provide a particularly relevant cell type for analysis of the response to hypoxia, because they demonstrate robust viability and even produce neurotrophic cytokines under hypoxia (12). We have purified and cloned an ORP with a molecular mass of $150 \mathrm{kDa}$ (termed ORP150) from primary cultures of astrocytes $(13,14)$. ORP150 is an inducible endoplasmic reticulum (ER) chaperone and is expressed in a range of pathologic situations, such as ischemic brain (15), atherosclerotic plaques (16), and malignant tumors (17). These data suggest that ORP150 may contribute in a fundamental way to the cellular response to environmental stress.

The current study demonstrates expression of ORP150 in healing of human wounds. In vivo genetransfer studies showed that infection of wounds with an adenovirus causing overexpression of ORP150 accelerated wound closure (and expression of VEGF antigen), whereas suppression of ORP150 delayed the reparative processes (and suppressed VEGF antigen expression). These findings suggest an as yet unexplored 
therapeutic target for modulation of angiogenesis, expression of the inducible ER chaperone ORP150.

\section{Methods}

Materials. For Western blot analysis, anti-human ORP150 Ab $(1 \mu \mathrm{g} / \mathrm{ml})(14)$, GRP78 $(0.2 \mu \mathrm{g} / \mathrm{ml}$; StressGen Biotechnologies Corp., Victoria, British Columbia, Canada), trans-Golgi network-38 (TGN38) (1 $\mu \mathrm{g} / \mathrm{ml}$; Transduction Laboratories, Lexington, Kentucky, USA), RAGE $(0.2 \mu \mathrm{g} / \mathrm{ml})$ (18), HSC70 (0.2 $\mu \mathrm{g} / \mathrm{ml}$; StressGen Biotechnologies Corp.), anti-KDEL $\mathrm{mAb}(0.2 \mu \mathrm{g} / \mathrm{ml}$; StressGen Biotechnologies Corp.), which recognizes GRP78 and GRP94, and anti-VEGF $\mathrm{Ab}(0.2 \mu \mathrm{g} / \mathrm{ml}$; Santa Cruz Biotechnology Inc., Santa Cruz, California, USA) were used. We used anti-human ORP150 IgG $(5 \mu \mathrm{g} / \mathrm{ml})$, anti-VEGF IgG $(1 \mu \mathrm{g} / \mathrm{ml}$; Santa Cruz Biotechnology Inc.), anti-CD68 IgG (5 $\mu \mathrm{g} / \mathrm{ml}$, DAKO Japan, Tokyo, Japan), or anti-plateletendothelial cell adhesion molecule-1/CD31 (PECAM1/CD31) IgG $(1 \mu \mathrm{g} / \mathrm{ml}$; Santa Cruz Biotechnology Inc.) for immunohistochemical analysis.

Cell culture and induction of bypoxia. Macrophages were prepared from human peripheral blood cells as described (19). In brief, monocytes were separated from human peripheral blood by density-gradient centrifugation (Histopaque 1077; Sigma Chemical Co. St. Louis, Missouri, USA) and cultured and differentiated for 7-10 days in RPMI-1640 containing 10\% human serum. The cultures were then exposed to hypoxia as described previously (20). Where indicated, cells were cultured either in the presence or in the absence of sodium nitroprusside (SNP), as described (21).

Immunohistochemical analysis of wounds and macrophages. Human wound samples were obtained from pathologic specimens under the auspices of an approved institutional review board protocol. Immunohistochemical analysis was performed as described previously (13).

Western blot analysis and immunoprecipitation. Western blot analysis was performed as described previously (22). Interaction of ORP150 and VEGF was assessed by immunoprecipitation using anti-ORP150 $\operatorname{IgG}(3 \mu \mathrm{g} / \mathrm{ml})$ and anti-VEGF IgG $(0.5 \mu \mathrm{g} / \mathrm{ml})$ as described (13).

Construction of adenovirus vectors. Replication-deficient recombinant adenovirus vector containing the cDNA for ORP150 was constructed according to the COS/TPC method as described (23). Briefly, the fulllength cDNA for rat ORP150 was subcloned in the sense or antisense orientation into the expression unit of pAxCAwt. The desired recombinant adenoviruses, generated by homologous recombination, were designated Ad/S-ORP150 (sense) and Ad/AS-ORP150 (antisense) and were grown in 293 cells and purified by cesium chloride gradient centrifugation followed by extensive dialysis.

RT-PCR. RT-PCR was performed as described (12). The following primers were derived from the human VEGF sequence: $5^{\prime}$-TTC TAC AAT GAG CTG CGT GTG GC$3^{\prime}, 5^{\prime}$-CTC ATA GCT CTT CTC CAG GGA GGA-3'. The amount of VEGF transcript was semiquantitatively assessed by comparison with the PCR product of either the $\beta$-actin or GAPDH transcript amplified simultaneously as an internal control $(12,24)$.

The fractionation of cultured human macrophages. The fractionation of cultured human macrophages was performed as described (25). In brief, human macrophages (about $10^{7}$ cells) were homogenized by nitrogen cavitation and centrifuged at 5,000 $g$ for 30 minutes at $4^{\circ} \mathrm{C}$. The supernatant was then subjected to ultracentrifugation $(100,000 \mathrm{~g})$ at $4^{\circ} \mathrm{C}$ for 3 hours in buffer containing HEPES $(20 \mathrm{mM}, \mathrm{pH} 7.4), \mathrm{KCl}$ (100 mM), $\mathrm{NaCl}(20 \mathrm{mM})$, EDTA $(5 \mathrm{mM}), \operatorname{PMSF}(1$ $\mathrm{mM})$, and a sucrose step gradient $(38,30$, and $20 \%)$. The pellet (fraction 1) was resuspended, the layered fractions (fractions 2-5) were collected, and each fraction was subjected to Western blot analysis using Ab's against markers specific for various cellular organelles, i.e., ER (GRP78), Golgi apparatus (TGN38), plasma membrane (RAGE), and cytosol (HSC70).

Determination of VEGF, $b F G F$, and TGF- $\beta 1$. Elaboration of VEGF, bFGF, and TGF- $\beta 1$ antigen was assessed using a commercially available kit (R\&D Systems Inc., Minneapolis, Minnesota, USA) according to the manufacturer's instructions. To assess VEGF antigen in the cell supernatant, cultured human macrophages $\left(2 \times 10^{5}\right.$ cells) were cultured in serumfree medium. To assess VEGF antigen in the ER fractions, fractions were also diluted in PBS $(0.2 \mathrm{ml})$ containing Nonidet P-40 (0.5\%), followed by the determination of VEGF antigen by ELISA as described above. The protein content was measured using the method described by Lowry (26).

Secondary intention wound-healing model. Full-thickness excisional skin wounds using 6-mm skin biopsy punches were made on the backs of 8-week-old C57BL/6J (wild-type mice) or 8-week-old C57BL/6J$\mathrm{m}^{+/+}$Leprdb homozygous mice ( $d b / d b$ mice) (both obtained from the Jackson Laboratory, Bar Harbor, Maine, USA), as described (27). For the evaluation of efficiency of adenovirus infection in wounds, the indicated amount of Ad/S-ORP150, Ad/AS-ORP150, AxCALacZ (28), or AxGFP (green fluorescent protein), provided by Riken (The Institute of Physical and Chemical Research, Tokyo, Japan) was diluted in PBS $(0.2 \mathrm{ml})$ and administered subcutaneously at each square $2 \mathrm{~mm}$ from the wound edge 2 days after the injury, using a Hamilton gas-tight syringe with a $28 \mathrm{G}$ needle. Wound tissue was dissected using $10-\mathrm{mm}$ skin biopsy punches 5 days after the injury. The number of cells positive for $\mathrm{F} 4 / 80$ in granulation tissue and the ratio of GFP-positive cells to F4/80-positive cells were counted by two investigators blinded to the experimental protocol. The tissue content of ORP150 was determined by ELISA analysis as described (29).

To assess the role of ORP150 in wound healing, skin wounds of the indicated size ( 6 or $12 \mathrm{~mm}$ ) were made on the backs of 8-week-old wild-type or 8-week-old $d b / d b$ mice as described above. The wound was digitally photographed, and the wound area was calculat- 
a

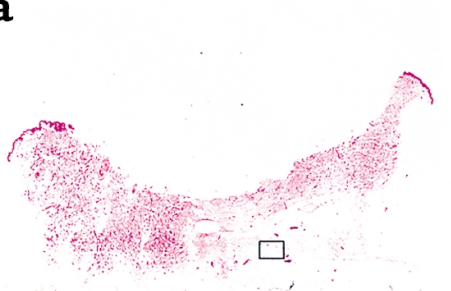

$H \& E$

\section{$\triangle \Delta$}

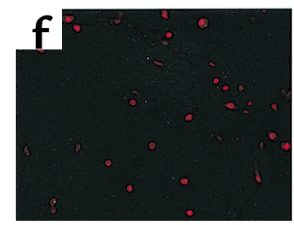

ORP150

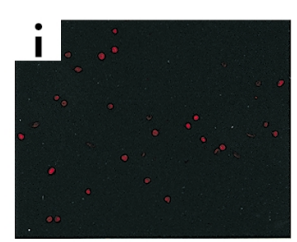

ORP150
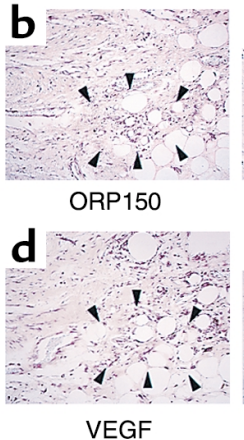

VEGF

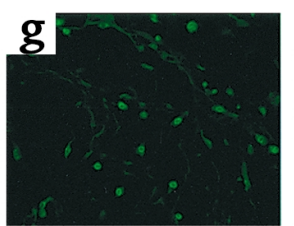

CD68

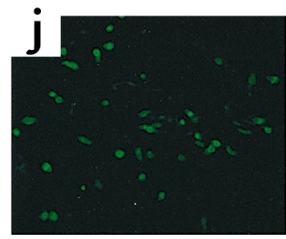

VEGF
C

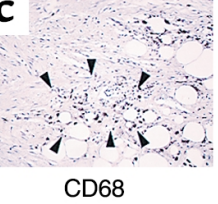

e
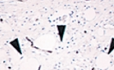

41

CD31

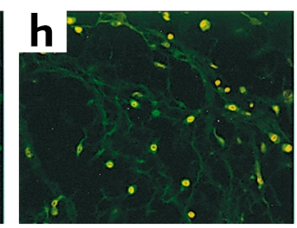

ORP150 + CD68

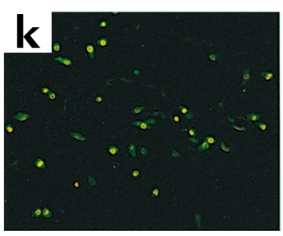

ORP150 + VEGF
Figure 1

ORP150 expression in healing human wound. (a-e) A human wound (31-year-old male) was analyzed either by $\mathrm{H} \& \mathrm{E}$ staining $(\times 2)$ (the area indicated by open box was further studied in $\mathbf{b}-\mathbf{k}$ ) or immunohistochemically using anti-ORP150 (×40) (b), anti-CD68 Ab $(\times 40)$ (c), anti-VEGF Ab $(\times 40)(\mathbf{d})$, or anti-CD31 Ab $(\times 40)$ (e), followed by visualization with the peroxidase method. (f-h) Sections adjacent to $\mathbf{b}$ (areas indicated by filled arrowheads) were double-stained with antiORP150 Ab (f) and anti-CD68 Ab (g). Both images were digitally overlapped $(\times 100)(\mathbf{h})$. (i-k) Sections adjacent to $\mathbf{f}$ were double-stained with anti-ORP150 $A b(\mathbf{i})$ and anti-VEGF Ab (j). Both images were digitally overlapped $(\times 100)(\mathbf{k})$. Open arrowheads in a denote the edge of the granulation tissue. See text for the description of filled arrowheads in $\mathbf{b}-\mathbf{e}$. ed using PhotoShop software (Adobe Systems Inc., Tokyo, Japan). After fixation, the wound tissue was cut in $5-\mu \mathrm{m}$ sections, frozen, and then stained with hematoxylin and eosin (H\&E) or Masson's trichrome. Each slide was assigned a histologic score ranging from 1 to 12 as described (27). Briefly, the scoring was based on the degree of the cellular invasion, granulation tissue formation, vascularity, and re-epithelialization. To assess the extracellular VEGF antigen of wound tissue, the tissues were homogenized with PBS, centrifuged at $2000 \mathrm{~g}$, and the supernatant was then subjected to ELISA as described above. For the evaluation of neovascularization during wound healing, the density of vascular structures was determined by immunostaining with anti-PECAM-1/CD31 IgG $(1 \mu \mathrm{g} / \mathrm{ml})$ (30), followed by quantitative analysis. Briefly, each slide was digitally photographed and captured using PhotoShop software. Using a freehand drawing tool, the wound bed was outlined, and the area of the wound bed was measured. The measurement of the PECAM-1-positive area within the wound bed was also performed. To assess the vessel density, the following formula was used: \% vascularization $=($ PECAM-1 - positive area $/$ total wound bed area) $\times 100$. These two assays were preformed by two investigators blinded to the experimental protocol.

Statistical analysis was performed by using either a nonpaired $t$ test or ANOVA followed by multiple comparison analysis. Where indicated, data were analyzed by two-way ANOVA followed by multiple contrast analysis.

\section{Results}

Expression of ORP150 in buman wounds. Immunohistochemical analysis of a human wound granular tissue was performed at 10 days after injury, as indicated by the open box in Figure 1a. Examination of the edge at higher magnification showed the accumulation of cells positive for ORP150, where the accumulation of CD68 signals, a marker for human macrophages (16), and VEGF signals was demonstrated in adjacent sections (Figure 1, b-d, indicated by the filled arrowheads). Scattered CD31 signal in the same area suggested the occurrence of neovascularization (Figure 1e). Furthermore, an immunofluorescence study demonstrated that ORP150 signals in the granulation tissue (Figure 1, $\mathrm{f}$ and i) overlapped, at least in part, with CD68 signals (Figure 1, $g$ and $h$ ) and VEGF signals (Figure $1, j$ and $k$ ). We have extended the immunohistochemical analysis with ten samples, which showed a similar tendency.

Role of ORP150 in the elaboration of VEGF by macrophages. In view of the known role of macrophages in neovascularization of wounds (31), we hypothesized that ORP150 might participate in cellular processing of secreted proteins, such as VEGF, released by macrophages under hypoxic conditions. Exposure of cultured macrophages to hypoxia $\left(\mathrm{pO}^{2} \approx 10\right.$ torr in the medium) resulted in time-dependent release of VEGF antigen into culture supernatants (Figure 2a), as well as an increase in VEGF transcripts (Figure 2b). Consistent with our previous observations (16), expression of ORP150 was also strongly enhanced in hypoxic macrophages (Figure 2c). These results led us to per- 
a

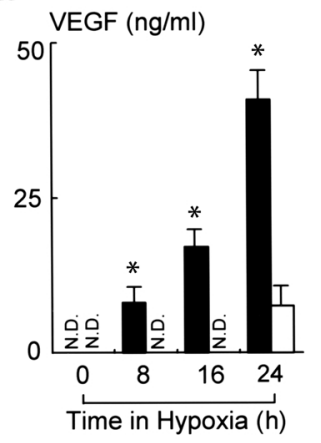

b

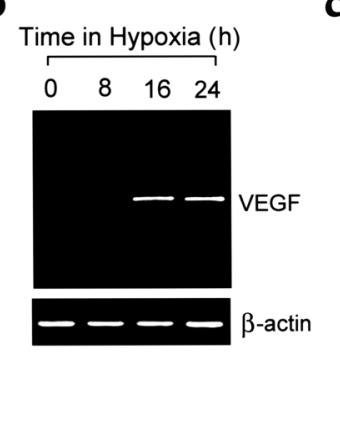

C

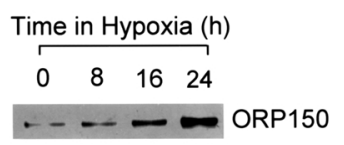

d

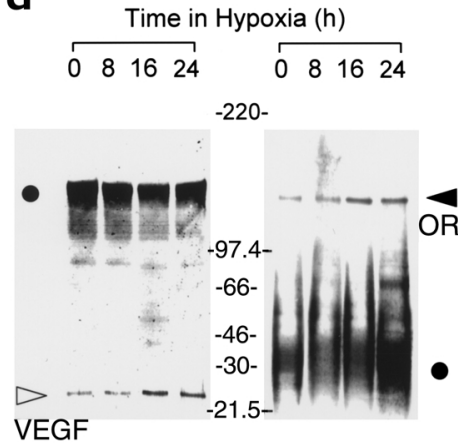

Figure 2

Expression of VEGF and ORP150 in human macrophages exposed to hypoxia. (a) Macrophages were either exposed to hypoxia (filled bars; 0-24 hours) or maintained under normoxic conditions (open bars). VEGF content in the culture supernatant was measured by ELISA as described in the text $(n=8$; mean $\pm \mathrm{SD})$. ${ }^{*} P<0.01$ by multiple contrast analysis. (b-d) Macrophages were exposed to hypoxia (0-24 hours). (b) RNA was prepared at the indicated times and subjected to RT-PCR analysis using primers for either VEGF (upper panel) or $\beta$-actin (lower panel). Protein extracts were prepared and subjected to Western blot analysis using anti-human ORP150 Ab (c). Cell lysates were incubated with either anti-human ORP150 Ab or anti-VEGF Ab. (d) The immunoprecipitant was separated in SDS-PAGE (8-12\%; nonreducing condition for VEGF and reducing condition for ORP150) and subjected to Western blot analysis using either anti-VEGF Ab (left panel) or anti-ORP150 Ab (right panel). Migrations of molecular-weight markers are shown in the middle. The filled circles denote the signals derived from IgG used as primary Ab. (e-g) Human macrophages were double-stained with anti-ORP150 Ab (e) and anti-VEGF Ab (f). VEGF and ORP150 signals were digitally overlapped (×400) (g). form immunoprecipitation analyses to determine if ORP150 and VEGF might interact; lysates of hypoxic macrophages were immunoprecipitated with antiORP150 IgG, and the immunoprecipitates were subjected to SDS-PAGE and immunoblotting with antiVEGF IgG. A prominent immunoreactive band was observed (VEGF; Figure 2d, left panel). Alternatively, when a complementary protocol was followed in which immunoprecipitation was performed with anti-VEGF IgG and immunoblotting employed anti-ORP150 IgG, an antigen of ORP150 was detected in precipitants (ORP150; Figure 2d, right panel). Studies in which either purified ORP150 or recombinant VEGF were immunoprecipitated with $\mathrm{Ab}$ to either VEGF or ORP150 demonstrated that anti-ORP150 does not react with VEGF and that anti-VEGF does not crossreact with ORP150 (not shown). Immunocytochemistry also showed ORP150 and VEGF to be present in an overlapping distribution within the cell (Figure 2, $\mathrm{e}-\mathrm{g}$ ), although VEGF immunoreactivity was also detected in the pericytoplasmic space (Figure 2, f and $\mathrm{g}$ ), while ORP150 is present in the ER (Figure 2e) (13). These data suggested that ORP150 binds to VEGF in the ER and may participate in protein transport as part of the secretory pathway.

To further assess the possibility of a role for ORP150 in the secretory pathway of VEGF, levels of ORP150 were modulated in macrophages using adenoviruses coding for ORP150 in the sense (Ad/S-ORP150) or antisense (Ad/AS-ORP150) orientation. The efficiency of viral infection into the cultured macrophages assessed using AxGFP (100 moi) was approximately equal to $80 \%$ in macrophages, although the efficiency was quite low in freshly prepared monocytes, as described previously $(32,33)$.

Treatment of human macrophages with Ad/ASORP150 suppressed hypoxia-mediated induction of ORP150 in macrophages, whereas treatment with Ad/SORP150 resulted in the increase of ORP150 (Figure 3a, upper panel). In contrast, treatment with an adenovirus overexpressing either LacZ (AxCALacZ) or GFP (AxGFP, 100 moi in each case) had no effect on ORP150 levels in hypoxic cultures (Figure 3a, upper panel). In each case, the levels of GRP78 and GRP94, other molecular chaperones in the ER, remain unchanged (Figure 3a, lower panels). Infection of hypoxic macrophages with adenoviruses encoding LacZ or ORP150 in the sense/antisense orientation (100 moi) had no effect on the level of VEGF, $\beta$-actin, or GAPDH transcripts (Figure $3 b$ ). ELISA analysis of VEGF in subcellular fractions (confirmed by Western blot analysis using markers specific to each organelle; Figure 3c) revealed an increase in VEGF content of the ER fraction in cultured macrophages subjected to hypoxia after infection with Ad/AS-ORP150, compared with that in cells infected with AxCALacZ (Figure 3d). Consistently, ELISA of VEGF antigen in the supernatant of hypoxic macrophages infected with various amounts of Ad/ASORP150 showed a dose-dependent decrease in the culture supernatant (approximately threefold; Figure 3g). 
The VEGF content in the ER showed no significant decrease by the infection of cultured macrophages with Ad/S-ORP150 (Figure 3e), however, infection of cultured macrophages with Ad/S-ORP150 increased the level of VEGF antigen in the culture supernatant (Figure $3 \mathrm{~h}$ ). To further clarify the role of ORP150 in the secretory pathway of VEGF in response to stimuli other than hypoxia, SNP, an NO liberator (21), was added to macrophages infected with AxCALacZ, Ad/S-ORP150, and Ad/AS-ORP150 (100 moi) (23). Under normoxic conditions, addition of SNP $(10 \mu \mathrm{M})$ elevated the levels of VEGF antigen in the supernatant (approximately equal to an eightfold increase), with no significant differences among cells infected with AxCALacZ, Ad/S-
ORP150, or Ad/AS-ORP150 (data not shown). In contrast, treatment with Ad/AS-ORP150 caused a marked retention of VEGF in ER under hypoxic conditions, especially in cultures stimulated by SNP (Figure 3f), with the decrease of VEGF elaboration (Figure 3i). Treatment with Ad/S-ORP150 resulted in the decrease of VEGF antigen in ER, with the marked increase in VEGF production (Figure 3, f and i), suggesting a role of ORP150 in the optimized secretion of VEGF under hypoxia. Under these conditions, treatment of hypoxic cultures with Ad/AS-ORP150 did not alter cell viability up to the longest time examined, 48 hours (not shown). Immunocytochemical analysis demonstrated that the localization of VEGF immunoreactivity was also a

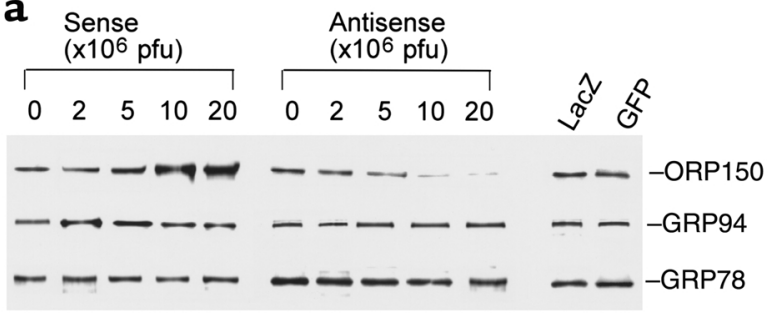

b
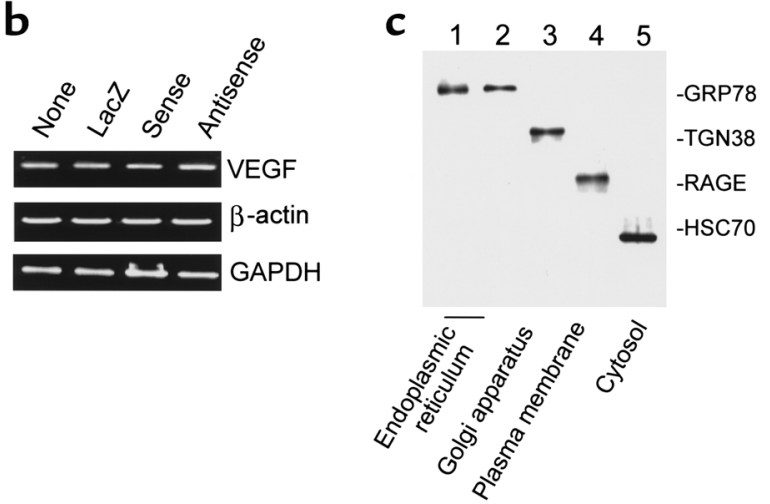

Figure 3

Dependence of VEGF processing on ORP150 in hypoxic macrophages. ( $\mathbf{a}$ and $\mathbf{b}$ ) Macrophages were exposed to hypoxia in the presence of Ad/S-ORP150, Ad/AS-ORP150, AxCALacZ, or AxGFP. Cell lysates were subjected to Western blot analysis using either anti-ORP150 Ab (a) (upper panel) or anti-KDEL $\mathrm{mAb}$ 's (lower panels). RNA was also prepared from cells incubated in the presence of adenovirus. (b) RT-PCR analysis was performed using primers specific for either VEGF (upper panel), $\beta$-actin (middle panel), or GAPDH (lower panel). (c) Cell lysates were fractionated, as described, and fractions corresponding to ER (lanes 1 and 2), Golgi apparatus (lane 3), plasma membrane (lane 4), and cytosol (lane 5) were subjected to immunoblot analysis using cellular organelle-specific Ab's. (d-i) Cultured macrophages were infected with AxCALacZ (100 moi), Ad/SORP150 (0-100 moi), or Ad/AS-ORP150 (0-100 moi), and further incubated under hypoxic conditions for 24 hours, either in the absence ( $\mathbf{d}, \mathbf{e}, \mathbf{g}$, and $\mathbf{h})$ or presence $(\mathbf{f}$ and $\mathbf{i})$ of SNP. The content of VEGF antigen in the ER fraction ( $\mathbf{d}-\mathbf{f})$ and culture supernatant (panels $\mathbf{g}$-i) was measured by ELISA as described $(n=6$; mean $\pm \mathrm{SD})$. ${ }^{*} P<0.01$ compared with AxCALacZ-treated culture by either multiple comparison analysis ( $\mathbf{d}, \mathbf{g}, \mathbf{h}$, and $\mathbf{f})$ or multiple contrast analysis, following two-way ANOVA (i). (j-o) Macrophages were infected with either AxCALacZ (j-I) or Ad/AS-ORP150 (m-o) and immunostained with either anti-KDEL mAb (j and $\mathbf{m})$ or anti-VEGF mAb. (I and o) Both images were digitally overlapped. $\times 400$.
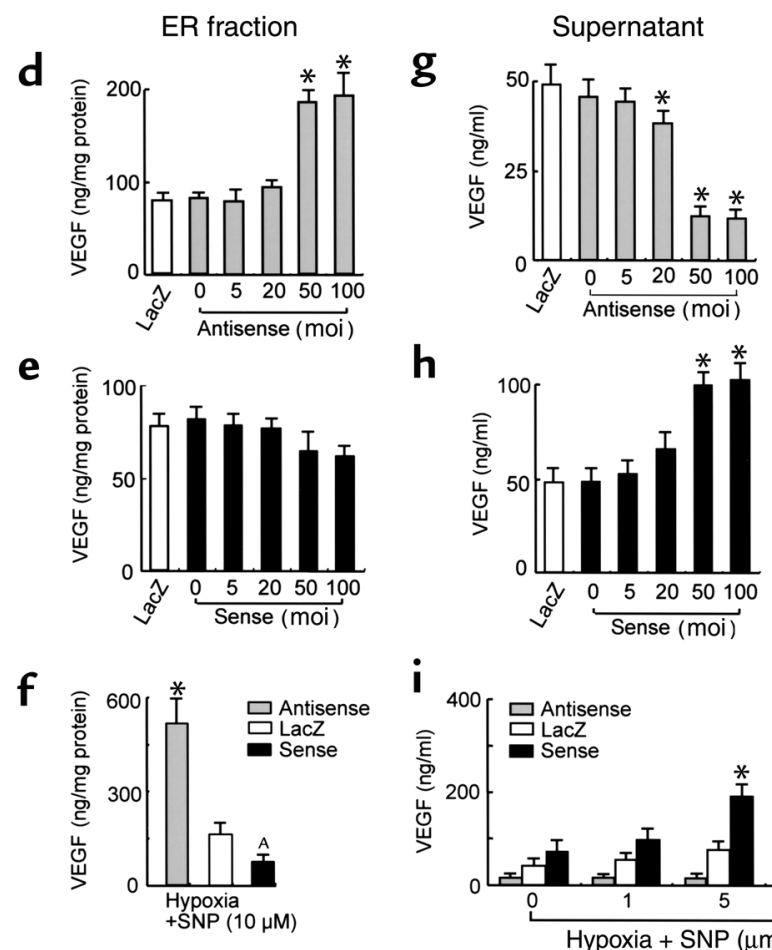

h

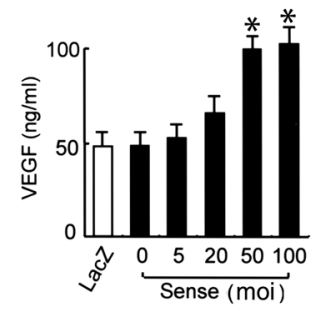

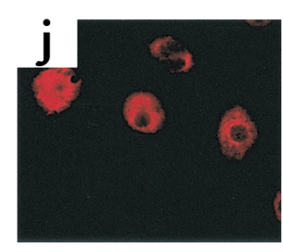

KDEL

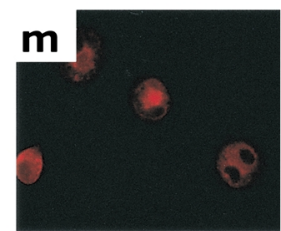

KDEL

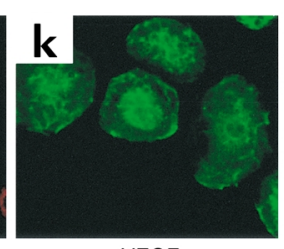

VEGF

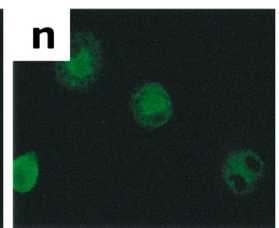

VEGF

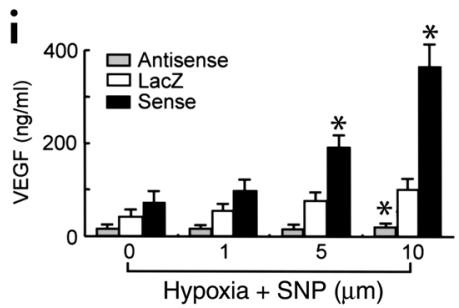

Hypoxia + SNP $(\mu \mathrm{m})$

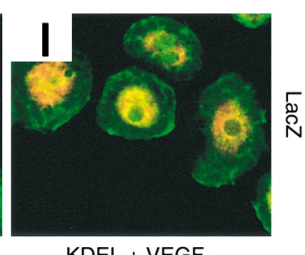

KDEL + VEGF

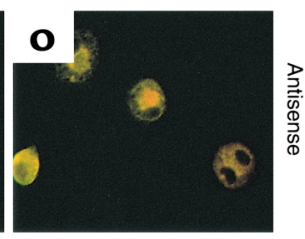

KDEL + VEGF 

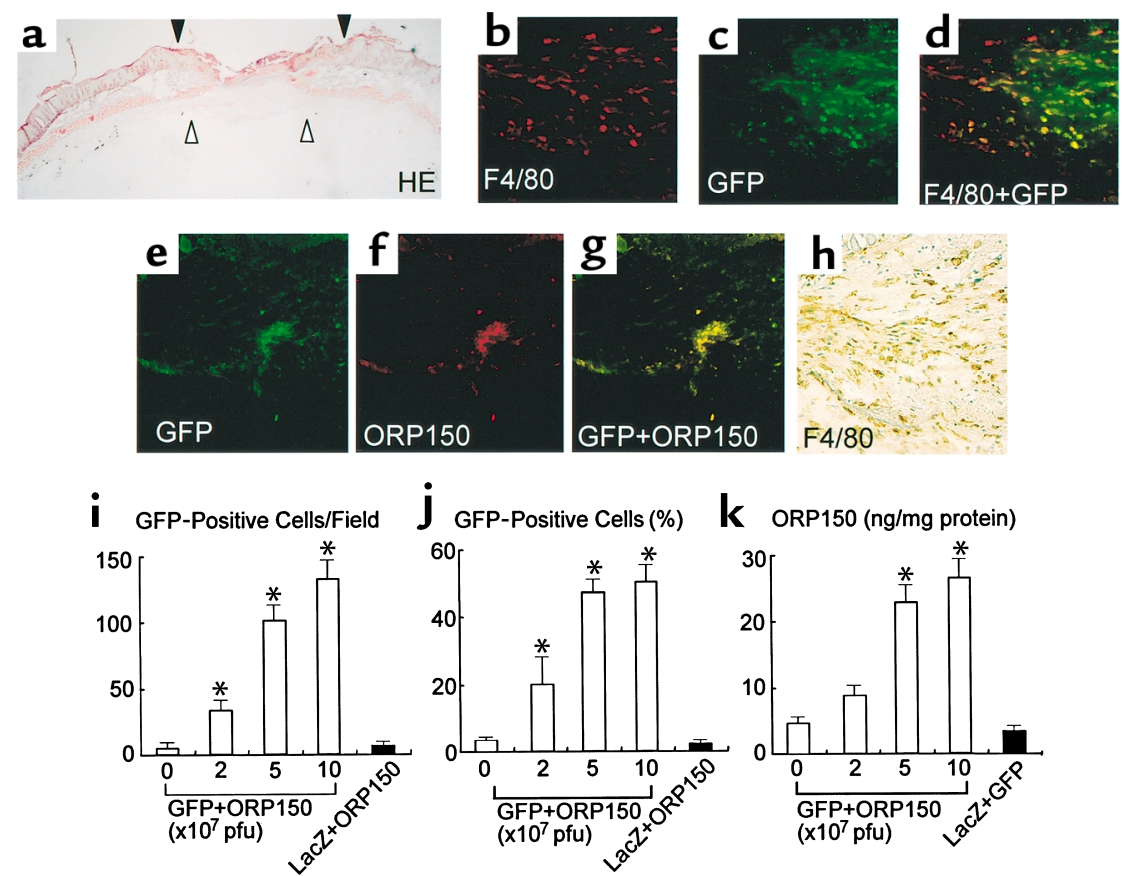

\begin{abstract}
Figure 4
Characterization of wounds infected with adenovirus. (a-h) Two days after the introduction of a wound (6 mm in diameter) in $d b / d b$ mice, a mixture of adenovirus (AxGFP and Ad/S-ORP150; $5 \times 10^{7}$ pfu each) was administered at each wound, as described. Two days after the infection, the tissue was sampled and subjected to H\&E staining $(\times 4)(\mathbf{a})$, immunostaining with anti-F4/80 Ab $(\times 40)(\mathbf{b})$, or visualization of GFP signal by fluorescent microscopy $(\times 40)(\mathbf{c})$. Signals derived from F4/80 and GFP were digitally overlapped $(\times 40)$ (d). (a) Filled arrowheads indicate the initial wound area, and the open arrowheads indicate the edge of the granulation tissue. (e-h) Granulation tissue was stained with anti-ORP150 Ab and signals derived from (e) GFP $(\times 40)$ and (f) ORP150 (×40) were visualized by fluorescent microscopy. (g) Signals of GFP and ORP150 were digitally overlapped. $(\times 40$.) (h) The adjacent section was immunostained with anti-F4/80 Ab. $(\times 40$.) (i-k) Either the indicated amount of AxGFP and Ad/S-ORP150 (open bars) or (i and j) AxCALacZ and Ad/SORP150 (108 pfu each; filled bars), or (k) AxCALacZ and AxGFP $\left(10^{8}\right.$ pfu each; filled bar) was administered, as described. Three days after the infection, either GFP-positive cells (i) or the percentage of GFP-positive cells in F4/80-positive cells (j) was counted in the granulation tissue as described in the text. The content of tissue ORP150 antigen was assessed by ELISA as described. (k). For $\mathbf{i}-\mathbf{k}, n=6$, mean $\pm \mathrm{SD} .{ }^{*} P<0.01$ by multiple comparison analysis.
\end{abstract}

observed in the pericytoplasmic space in macrophages infected with AxCALacZ (Figure 3, $j-1$ ), whereas the localization of VEGF immunoreactivity was observed only in the perinuclear space, which overlapped the signals obtained from anti-KDEL $\mathrm{Ab}$, in hypoxic macrophages infected with Ad/AS-ORP150 (Figure 3, $\mathrm{m}-\mathrm{o})$. These data indicate that ORP150 mediates the intracellular transport of VEGF in human macrophages, especially under hypoxic conditions.

Role of ORP150 in wound healing. To assess the efficiency of adenovirus in mouse wounds, a series of histochemical analyses were performed in $d b / d b$ mice after infection with recombinant adenovirus vector (Figure 4a; H\&E staining is shown for orientation). First, fluorescent microscopic analysis revealed an overlap of cells positive for $\mathrm{F} 4 / 80$ (specific for murine macrophages) and GFP-positive cells in granulation tissue of mouse wounds (Figure 4, b-d). The latter increased in parallel with the titer of coinfected adenovirus (Ad/SORP150 and AxGFP), whereas coinfection with Ad/S-ORP150 and AxCALacZ did not induce fluorescent cells (Figure 4i). The number of GFP-positive cells reached a maximum 2-3 days after the viral infec- tion, and signals could be detected up to 10 days after the viral infection (data not shown). Second, statistical analysis using $\mathrm{F} 4 / 80$ as a marker revealed the increase of overlap of GFP-positive cells with macrophages in a dose-dependent manner, showing maximal overlap (about $50 \%$ ) at $10^{8}$ pfu (Figure $4 \mathrm{j}$ ), though there were far less positive GFP signals derived from keratinocytes (data not shown). Third, coinfection of AxGFP and Ad/S-ORP150 showed the colocalization of signals derived from GFP fluorescence and ORP150 antigen (Figure 4, e-g), which, at least in part, overlapped with signals of F4/80 (Figure 4h). The increase in ORP150 antigen induced by adenovirus infection occurred in a dose-dependent manner (Figure $4 \mathrm{k})$. By contrast, coinfection of AxCALacZ and AxGFP failed to increase ORP150 antigen level in tissue. These data suggest that adenovirus infection occurred mainly in macrophages during wound healing, and the efficiency of infection was maximal (about $50 \%$ using GFP as a marker) with $0.5 \times 10^{8}$ to $1 \times 10^{8}$ pfu. Consequently, further experiments were carried out with $5 \times 10^{7} \mathrm{pfu}$ and $2 \times 10^{8} \mathrm{pfu}$ for $6-\mathrm{mm}$ and 12 mm wounds, respectively. 
To examine the effect of ORP150 on wound healing, a secondary intention wound-healing model was employed in genetically diabetic mice $\left(\mathrm{C} 57 \mathrm{BL} / 6 \mathrm{~J}-\mathrm{m}^{+/+}\right.$ $L_{e p r^{d b}}$ homozygous mice). Because this model has been used to study the effects of growth/angiogenic factors, we know that the reparative response is delayed and histologic scoring of these wounds shows diminished epithelialization $(27,34)$. Consistent with these previous observations, wound repair was delayed in C57BL/6J-m ${ }^{+/+}$Lepr $^{d b}$ homozygous mice $(d b / d b)$ administered AxCALacZ, with only about $40 \%$ closure by days 9-12 (Figure 5, a and b, open bars) and low histologic scores (Figure $5 \mathrm{c}$, open bars), whereas heterozygous $(d b /-)$ mice were virtually completely healed by this time (not shown). We hypothesized that overexpression of ORP150 in diabetic wounds, using an adenovirus strategy (Ad/S-ORP150), would accelerate wound repair. Topical treatment of $d b / d b$ animals with the same titer of Ad/S-ORP150 as was used for AxCALacZ controls resulted in accelerated wound closure (Figure 5, a and $\mathrm{b}$, filled bars) and an increase in histologic score (Figure $5 \mathrm{c}$ ), approximating that which was observed in control animals. By the same logic we reasoned that suppression of ORP150 would attenuate wound repair in C57BL/6J (wild-type) mice. Local administration of Ad/AS-ORP150 delayed the wound repair (Figure 5, d and e, filled bars) and caused a decrease in histologic score (Figure 5f). The administration of an inert virus had an effect on wound repair similar to that observed with AxCALacZ (data not shown).
Contribution of ORP150 to neovessel formation in wounds. To study the role of ORP150 expression on wound healing in further detail, we performed immunoblot analysis on murine wound tissue after infection of wounds with the different adenoviruses, this time using wounds of a smaller size (diameter $6 \mathrm{~mm}$ ) to facilitate quantitative analysis of vascularization and VEGF content. In diabetic mice, local administration of Ad/S-ORP150 accelerated the wound healing (Figure 6a), along with approximately fourfold increased levels of ORP150 antigen (Figure 6b) compared with wound tissue infected with AxCALacZ, as assessed by the densitometric analysis (Figure 6c). Immunohistochemical analysis demonstrated the increased expression of ORP150 led to angiogenic foci by day 6 after wounding (not shown). In parallel, increased ORP150 expression in diabetic wounds caused the increase of neovessel-occupied area in granulation tissue of $d b / d b$ mice treated with Ad/S-ORP150, compared with those receiving the AxCALacZ virus (Figure $6 \mathrm{~d}$ ), as well as the marked increase in tissue levels of extracellular VEGF by day 6 (Figure 6e).

To further confirm the role of ORP150 in wound healing, counterpart experiments were performed in wildtype mice. In wild-type mice, administration of Ad/ASORP150 delayed wound healing (Figure 6f), and wound tissue treated with Ad/AS-ORP150 displayed reduced ORP150 antigen up to day 6 , compared with wound tissue treated with AxCALacZ virus (Figure 6, g and h). Local administration of Ad/AS-ORP150 in wounds of
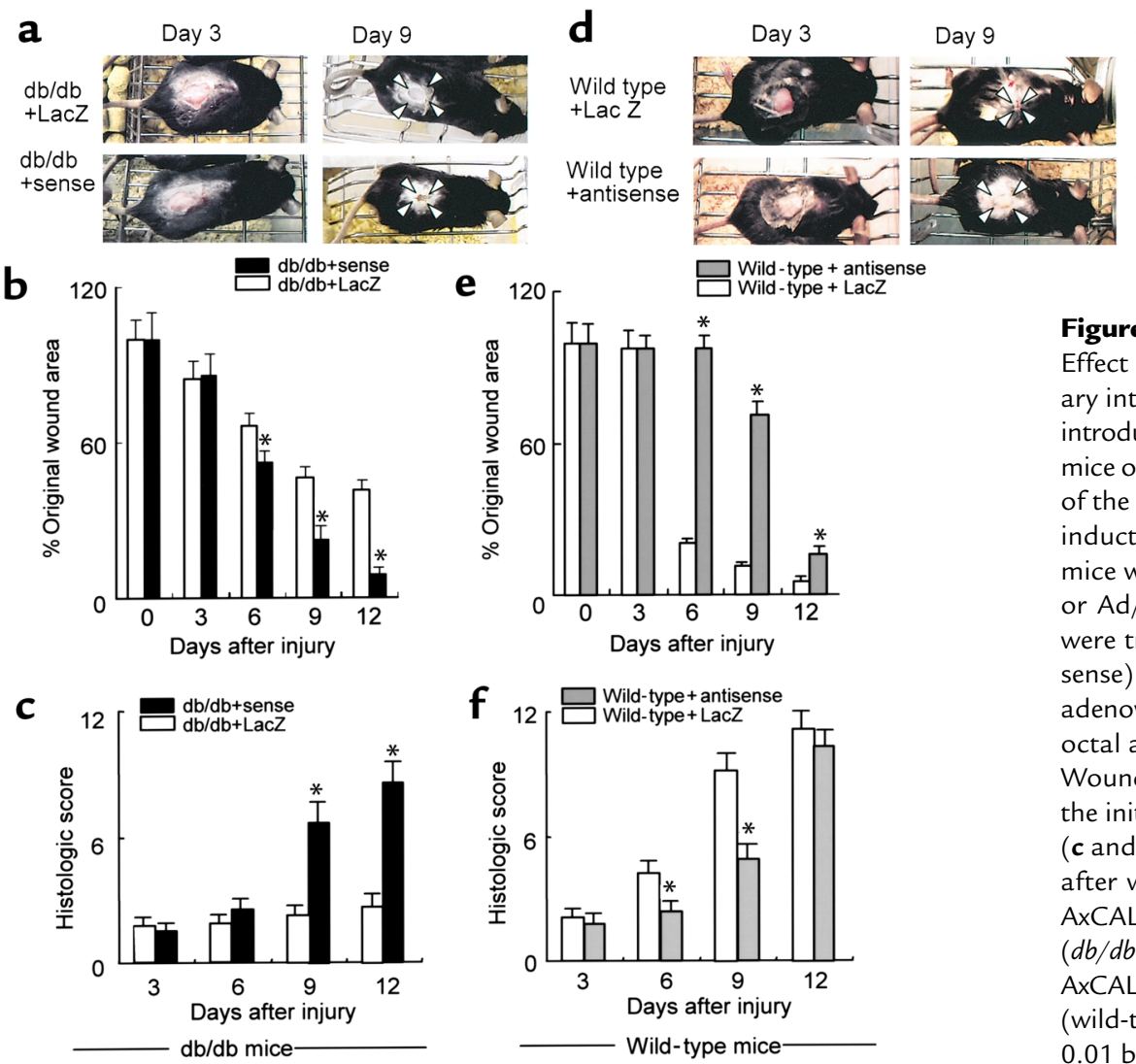
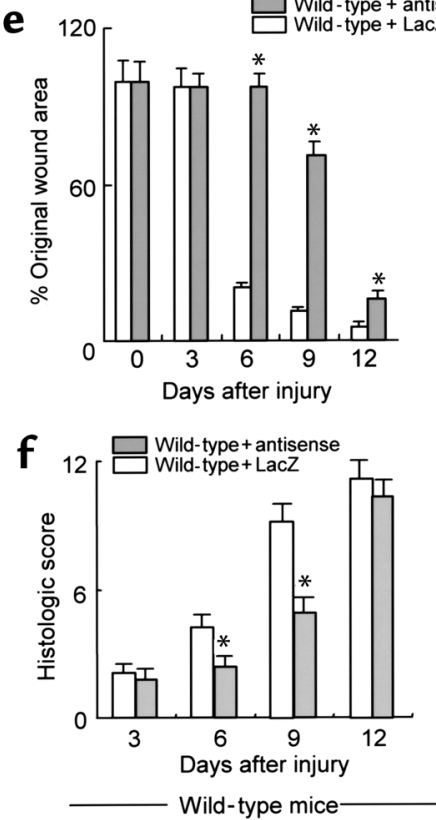

\section{Figure 5}

Effect of ORP150 on wound closure. A secondary intention wound (12 $\mathrm{mm}$ in diameter) was introduced in either C57BL/6J-m ${ }^{+/+} \operatorname{Lepr}^{d b}(\mathrm{db} / \mathrm{db})$ mice or C57BL/6J (wild-type) mice. Photographs of the wounds are shown on days 3 and 9 after induction of the wounds ( $\mathbf{a}$ and $\mathbf{d}$ ). The $d b / d b$ mice were treated with either AxCALacZ ( $L a c Z$ ) or Ad/S-ORP150 (sense), and wild-type mice were treated with either Ad/AS-ORP150 (antisense) or AxCALacZ. In each case, $2 \times 10^{8}$ pfu of adenovirus was subcutaneously injected at each octal angle of the wound $2 \mathrm{~mm}$ from the edge. Wound closure, shown as percentage of area of the initial wound ( $\mathbf{b}$ and $\mathbf{e}$ ) and histologic score (c and $\mathbf{f}$ ) were determined on the indicated day after wounding: $d b / d b$ mice were treated with AxCALacZ $(d b / d b+$ LacZ) or Ad/S-ORP150 $(d b / d b+$ sense $)$, and wild-type were treated with AxCALacZ (wild-type + LacZ) or Ad/AS-ORP150 (wild-type + antisense). $n=4, ;$ mean \pm SD. ${ }^{*} P<$ 0.01 by multiple contrast analysis. 

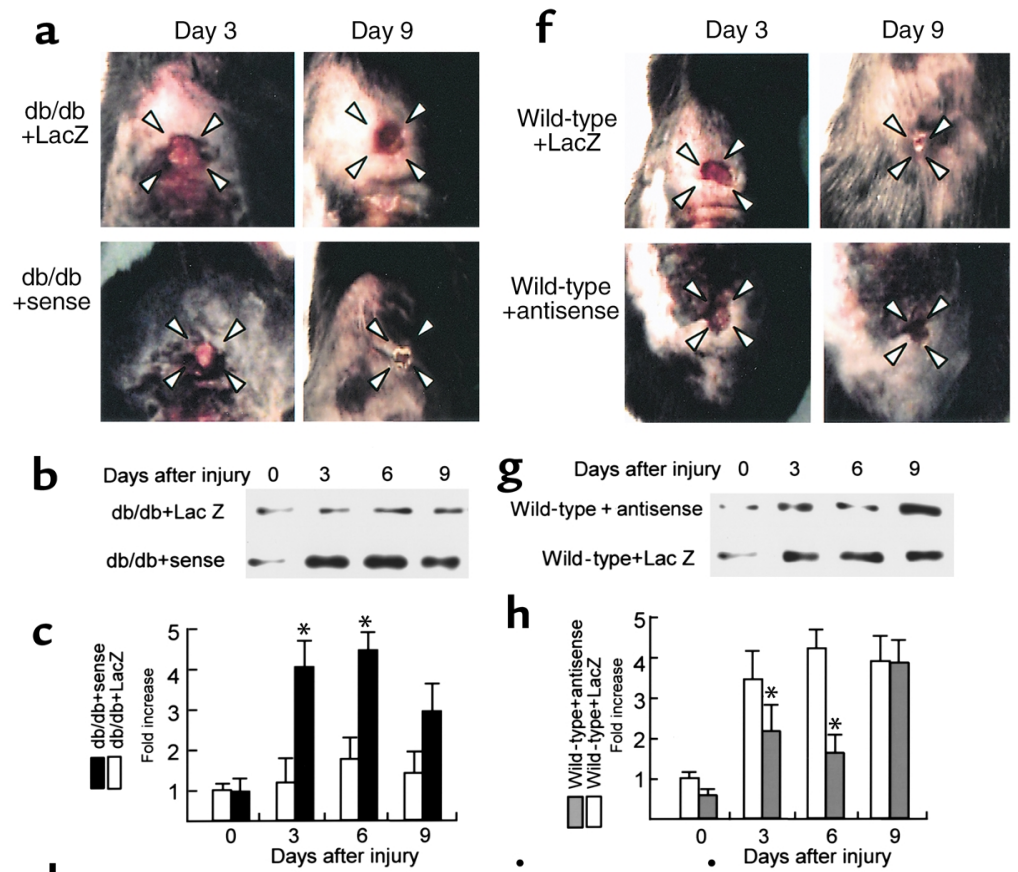

$\mathbf{h}$
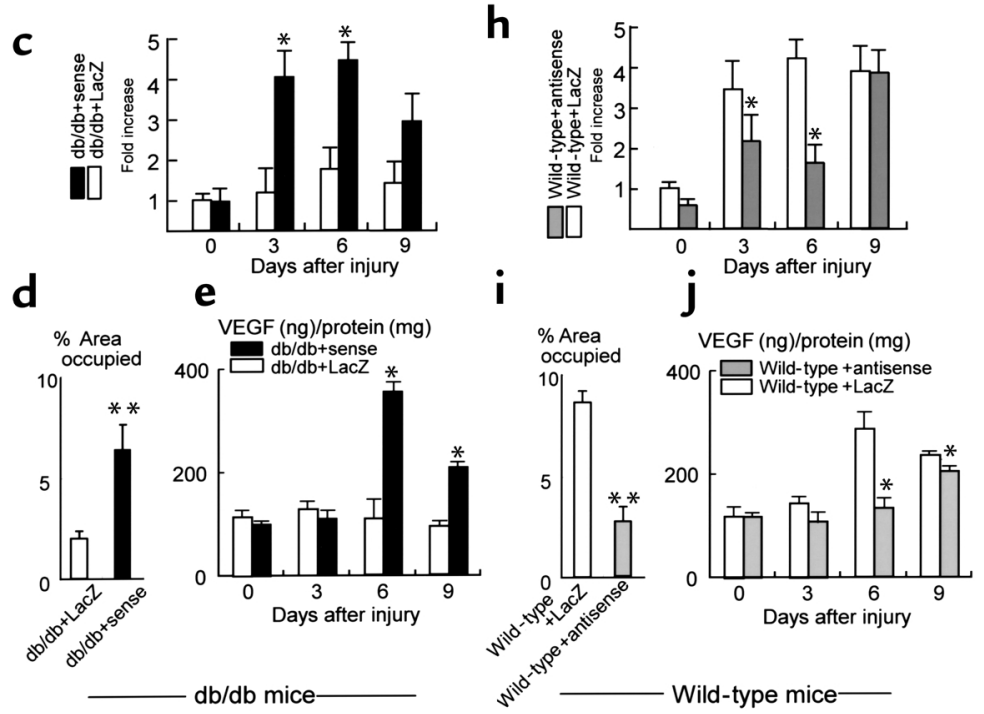

\section{Figure 6}

ORP150 content in wounds after infection with recombinant adenoviruses. A secondary intention wound model ( $6 \mathrm{~mm}$ in diameter) was introduced in C57BL/6J-m $\mathrm{m}^{+/+}$Lepr $^{d b}$ homozygous mice $(d b / d b)$ (a-e) and C57BL/6J mice (wild-type) $(\mathbf{f}-\mathbf{j})$. Two days after the injury, recombinant adenovirus $(5 \times$ $10^{7}$ pfu each) was administered as described above. Photographs of the wounds are shown on days 3 and 9 after induction of the wounds ( $\mathbf{a}$ and $\mathbf{f}$ ). At the indicated time points, wounds were sampled (10 $\mathrm{mm}$ diameter), and protein extracts were prepared from the wound tissue and subjected to Western blot analysis with anti-ORP150 Ab. A typical example of four repeated experiments is shown (b and $\mathbf{g})$. Densitometric analysis of multiple Western blots was performed in (c) $(d b / d b$ mice) and (h) (wild-type mice). ${ }^{*} P<0.01$ by multiple contrast analysis. ( $\mathbf{d}$ and $\mathbf{i}$ ) Angiogenesis was estimated semiquantitatively 7 days after the injury based on the area occupied by PECAM- 1 immunoreactive tissue. $n=6$; mean $\pm \mathrm{SD}$. ${ }^{*} P<0.01$ by nonpaired $t$ test. (e and $\mathbf{j}$ ) Tissue VEGF content was measured in wound tissue. $n=6$; mean \pm SD. ${ }^{*} P<0.01$ by multiple contrast analysis. Statistics were performed in comparison with animals infected with AxCALacZ (open bars) in each panel.

wild-type mice showed delayed granulation (not shown) and angiogenesis (Figure 6i), along with suppression of extracellular VEGF antigen by day 6 (Figure 6j).

To examine the effect of other factors on wound healing, tissue contents of bFGF and TGF- $\beta 1$ were assessed in the same way as for VEGF in both $d b / d b$ mice and wild-type mice, and no significant changes of these factors were detected in $d b / d b$ mice treated with either Ad/S-ORP150 or AxCALacZ or in wild-type mice treated with either Ad/AS-ORP150 or AxCALacZ (data not shown). In both experiments, number of CD68-positive cells showed no significant difference between wound tissue infected with Ad/S-ORP150, Ad/AS-ORP150, or AxCALacZ (data not shown).

\section{Discussion}

One of the salient features of the cellular biosynthetic response to hypoxia is redirection of protein synthesis with production of ORPs, which overlap with glucoseregulated proteins (GRPs) $(11,14,35,36)$. Common denominators of this group of polypeptides include their placement in the heat-shock protein family, the presence of ER retention sequences, and their properties as molecular chaperones in the ER-to-Golgi transfer of newly synthesized proteins (37). Since
ORPs/GRPs are induced by hypoxic stress in a wide range of tissues, their expression indicates that cellular components in wounds execute a stress response to the insufficient blood supply. Colocalization of ORP150 with VEGF found at macrophages in human wounds (Figure 1) suggests that these two molecules contribute in a complementary manner to the evolving stress response, at least in an early step in a series of reactions to achieve wound healing.

Since VEGF requires posttranslational processing (38-40), we considered the possibility that in the setting of cellular stress imposed by hypoxia, optimal function of molecular chaperones would be essential for secretion of mature VEGF. Our current observations demonstrate binding of ORP150 to VEGF in hypoxic macrophages. Suppression of ORP150 in hypoxic macrophages inhibited elaboration of VEGF, resulting in retention of this peptide in the ER. This is consistent with our previous observation that ORP150 binds to the ER form of the $80-\mathrm{kDa}$ glycoprotein (GP80), a major secretory protein in Madin-Darley canine kidney cells, and provides a more efficient pathway for protein transport due to its higher affinity for ATP than that of GRP78 (41). In addition, we have found that ORP150 enhanced elaboration of neu- 
rotrophic peptide in cultured neurons exposed to hypoxia and in an ischemic brain (42). These data suggest that ORP150 functions as molecular chaperone under hypoxia to facilitate the protein transport/processing in ER, though not specific to VEGF.

In the wound-healing process, macrophages infiltrating the wound site produce and secrete various kinds of cytokines or growth factors (2). TGF- $\beta 1$ and bFGF are known to be important factors secreted by macrophages in healing wounds; however, their levels showed no significant change in the wound tissues infected with adenovirus overexpressing ORP150 sense or antisense orientation mRNA. In contrast, while not affected by the levels of ORP150 in normoxia, SNPinduced VEGF production was much enhanced by the overexpression of ORP150 under hypoxic condition. Since nitric oxide (NO) liberator is known to suppress hypoxia-mediated induction of VEGF at the transcription level (43), it is possible that the beneficial effect of ORP150 functions mainly on posttranslational processing/export in the ER under hypoxia.

In diabetic wounds, in which one key facet of impaired repair is diminished evidence of vascular ingrowth (Figure 6d), we found decreased levels of ORP150 (Figure 6, b and c), because impaired stress response has been observed in the diabetic condition (44). Though the efficiency of infection was lower than the previous report (45), overexpression of ORP150 in wounds of diabetic mice had a beneficial effect. This is probably because even with only a limited number of cells displaying increased levels of ORP150 as a consequence of the viral infection procedure, their production of paracrine factors, such as VEGF, changed the course of wound repair by impacting on many cells in the local environment. As shown in Figure 3i, in cultured cells, the suppression of ORP150 is crucial for VEGF elaboration (by about 20 times suppression), especially when other factors exist that promote the VEGF production at the transcriptional level (e.g., NO or other cytokines). In contrast, manipulation of the ORP150 level in vivo resulted in modification of tissue VEGF content to only a small extent (twofold to threefold increase in diabetic wounds, for instance). The infection efficiency of adenovirus and the numbers of copies of cDNA introduced in tissue (Figure 4) would be less than those observed in in vivo experiments (Figure 3), which resulted in the small modification of VEGF secretion in wounds even with the existence of many factors functioning to boost the elaboration of VEGF. However, the data demonstrated here would at least claim that the optimization of protein transport by the expression of molecular chaperons could be a novel therapeutic target.

Taken together, these data highlight the role of ER chaperones in the cellular response to stress and suggest a new level of intervention, bolstering expression of ORP150 in settings such as impaired wound repair where protein processing events in the ER may limit the pace and completeness of repair.

\section{Acknowledgments}

We sincerely thank T. Tamatani (Department of Neuroanatomy), R. Mori (Department of Legal Medicine), and Y. Ishida (Department of Legal Medicine) at Kanazawa University for excellent technical assistance.

1. Ferrara, N., et al. 1996. Heterozygous embryonic lethality induced by targeted inactivation of the VEGF gene. Nature. 380:439-442.

2. Singer, A.J., and Clark, R.A. 1999. Cutaneous wound healing. N. Engl. J. Med. 341:738-746.

3. Larcher, F., et al. 1996. Up-regulation of vascular endothelial growth factor/vascular permeability factor in mouse skin carcinogenesis correlates with malignant progression state and activated $\mathrm{H}$-ras expression levels. Cancer Res. 56:5391-5396.

4. Machein, M.R., Risau, W., and Plate, K.H. 1999. Antiangiogenic gene therapy in a rat glioma model using a dominant-negative vascular endothelial growth factor receptor 2. Hum. Gene Ther. 10:1117-1128.

5. Alvarez Arroyo, M.V., et al. 1998. Role of vascular endothelial growth factor in the response to vessel injury. Kidney Int. Suppl. 68:S7-S9.

6. Jakeman, L.B., Winer, J., Bennett, G.L., Altar, C.A., and Ferrara, N. 1992. Binding sites for vascular endothelial growth factor are localized on endothelial cells in adult rat tissues. J. Clin. Invest. 89:244-253.

7. Plate, K.H., Breier, G., Weich, H.A., and Risau, W. 1992. Vascular endothelial growth factor is a potential tumour angiogenesis factor in human gliomas in vivo. Nature. 359:845-848.

8. Stone, J., et al. 1995. Development of retinal vasculature is mediated by hypoxia-induced vascular endothelial growth factor (VEGF) expression by neuroglia. J. Neurosci. 15:4738-4747.

9. Ladoux, A., and Frelin, C. 1993. Hypoxia is a strong inducer of vascular endothelial growth factor mRNA expression in the heart. Biochem. Biophys. Res. Commun. 195:1005-1010.

10. Kuwabara, K., et al. 1995. Hypoxia-mediated induction of acidic/basic fibroblast growth factor and platelet-derived growth factor in mononuclear phagocytes stimulates growth of hypoxic endothelial cells. Proc. Natl. Acad. Sci. USA. 92:4606-4610.

11. Heacock, C.S., and Sutherland, R.M. 1986. Induction characteristics of oxygen regulated protein. Int. J. Radiat. Oncol. Biol. Phys. 12:1287-1290.

12. Maeda, Y., et al. 1994. Hypoxia-reoxygenation mediated induction of interleukin- 6 in cultured rat astrocytes and expression in ischemic gerbil brain: a paracrine mechanism enhancing neuron-survival. J. Exp. Med. 180:2297-2308.

13. Kuwabara, K., et al. 1996. Purification and characterization of a novel stress protein, the $150 \mathrm{kDa}$ oxygen regulated protein (ORP150), from cultured rat astrocytes, and its expression in ischemic mouse brain. J. Biol. Chem. 279:5025-5032.

14. Ikeda, J., et al. 1997. Cloning and expression of cDNA encoding the human $150 \mathrm{kDa}$ oxygen regulated protein, ORP150. Biochem. Biophys. Res. Commun. 230:94-99.

15. Matsushita, K., et al. 1998. Marked, sustained expression of a novel 150$\mathrm{kDa}$ oxygen regulated stress protein, in severely ischemic mouse neurons. Mol. Brain Res. 60:98-106.

16. Tsukamoto, Y., et al. 1996. $150 \mathrm{kDa}$ oxygen regulated protein (ORP150) is expressed in human atherosclerotic plaques and allows mononuclear phagocytes to withstand cellular stress on exposure to hypoxi and modified LDL. J. Clin. Invest. 98:1930-1941.

17. Tsukamoto, Y., et al. 1998. Expression of $150 \mathrm{kDa}$ oxygen-regulated protein (ORP150), a new member of the HSP70 family, in human breast cancers. Lab. Invest. 78:699-706.

18. Yamaguchi, A., et al. 1999. Stress-associated endoplasmic reticulum protein 1 (SERP1)/ribosome-associated membrane protein 4 (RAMP4) stabilizes membrane proteins during stress and facilitates subsequent glycosylation. J. Cell Biol. 147:1195-1204.

19. Koga, S., et al. 1992. Synthesis and release of interleukin-1 by reoxygenated human mononuclear phagocyte. J. Clin. Invest. 90:1007-1015.

20. Ogawa, S., et al. 1990. Hypoxia modulates the barrier and coagulant function of cultured bovine endothelium. J. Clin. Invest. 85:1090-1098.

21. Frank, S., Stallmeyer, B., Kampfer, H., Kolb, N., and Pfeilschifter, J. 1999. Nitric oxide triggers enhanced induction of vascular endothelial growth factor expression in cultured keratinocytes (HaCaT) and during cutaneous wound repair. FASEB J. 13:2002-2014.

22. Ozawa, K., et al. 1999. 150-kDa oxygen-regulated protein (ORP150) suppresses hypoxia-induced apoptotic cell death. J. Biol. Chem. 274:6397-6404.

23. Miyake, S., et al. 1996. Efficient generation of recombinant adenoviruses using adenovirus DNA-terminal protein complex and a cosmid bearing the full-length virus genome. Proc. Natl. Acad. Sci. USA. 93:1320-1324.

24. Matsuo, N., et al. 1995. Cloning of a novel RNA binding polypeptide (RA301) induced by hypoxia/reoxygenation. J. Biol. Chem. 270:28216-28222.

25. Yan, S.D., et al. 1999. Role of ERAB/L-3 hydroxyacyl-coenzyme A dehy- 
drogenase type II activity in A $\beta$-induced cytotoxicity. J. Biol. Chem. 274:2145-2156.

26. Lowry, O., Rosenbrough, N.J., Farr, L.A., and Randall, R.J. 1951. Protein measurement with the Folin phenol reagent. J. Biol. Chem. 193:265-275.

27. Greenhalgh, D.G., Sprugel, K.H., Murray, M.J., and Ross, R. 1990. PDGF and FGF stimulate wound healing in the genetically diabetic mouse. Am. J. Pathol. 136:1235-1246.

28. Kanegae, Y., et al. 1995. Efficient gene activation in mammalian cells by using recombinant adenovirus expressing site-specific Cre recombinase. Nucleic Acids Res. 23:3816-3821.

29. Brett, J., et al. 1993. Survey of the distribution of a newly characterized receptor for advanced glycation end products in tissues. Am. J. Pathol. 143:1699-1712.

30. Berman, M.E., Xie, Y., and Muller, W.A. 1996. Roles of platelet/endothelial cell adhesion molecule-1 (PECAM-1, CD31) in natural killer cell transendothelial migration and $\beta 2$ integrin activation. J. Immunol. 156:1515-1524.

31. Swift, M.E., Kleinman, H.K., and DiPietro, L.A. 1999. Impaired wound repair and delayed angiogenesis in aged mice. Lab. Invest. 12:1479-1487.

32. Bondeson, J., Browne, K.A., Brennan, F.M., Foxwell, B.M., and Feldmann, M. 1999. Selective regulation of cytokine induction by adenoviral gene transfer of I $\mathrm{I} B \alpha$ into human macrophages: lipopolysaccharide-induced, but not zymosan-induced, proinflammatory cytokines are inhibited, but IL-10 is nuclear factor- $\mathrm{KB}$ independent. J. Immunol. 162:2939-2945.

33. Foxwell, B., et al. 1998. Efficient adenoviral infection with $\mathrm{I} \kappa \mathrm{B} \alpha$ reveals that macrophage tumor necrosis factor- $\alpha$ production in rheumatoid arthritis is NF-KB dependent. Proc. Natl. Acad. Sci. USA. 95:8211-8215.

34. Brown, L.F., et al. 1992. Expression of vascular permeability factor (vascular endothelial growth factor) by epidermal keratinocytes during wound healing. J. Exp. Med. 176:1375-1379.

35. Lee, A.S. 1992. Mammalian stress response: induction of the glucose reg- ulated protein family. Curr. Opin. Cell Biol. 4:267-273.

36. Roll, D.E., Murphy, B.J., Laderoute, K.R., Sutherland, R.M., and Smith, H.C. 1991. Oxygen regulated $80 \mathrm{kDa}$ protein and glucose regulated $78 \mathrm{kDa}$ protein are identical. Mol. Cell. Biochem. 103:141-148.

37. Herrmann, J.M., Malkus, P., and Schekman, R. 1999. Out of the ER: outfitters, escorts and guides. Trends Cell Biol. 9:5-7.

38. Claffey, K.P., Senger, D.R., and Spiegelman, B.M. 1995. Structural requirements for dimerization, glycosylation, secretion, and biological function of VPF/VEGF. Biochim. Biophys. Acta. 1246:1-9.

39. Keck, R.G., Berleau, L., Harris, R., and Keyt, B.A. 1997. Disulfide structure of the heparin binding domain in vascular endothelial growth factor: characterization of posttranslational modifications in VEGF. Arch. Biochem. Biophys. 344:103-113.

40. Walter, D.H., et al. 1996. The in vivo bioactivity of vascular endothelial growth factor/vascular permeability factor is independent of $\mathrm{N}$-linked glycosylation. Lab. Invest. 74:546-556.

41. Bando, Y., et al. 2000. The $150 \mathrm{kDa}$ oxygen regulated protein (ORP150) functions as a novel molecular chaperone in the protein transport of the MDCK cells. Am. J. Physiol. Cell Physiol. 278:C1172-C1182.

42. Tamatani, M., et al. 2001. ORP150 protects against hypoxia/ischemiainduced neuronal death. Nat. Med. 7:317-323.

43. Tuder, R.M., Flook, B.E., and Voelkel, N.F. 1995. Increased gene expression for VEGF and the VEGF receptors KDR/Flk and Flt in lungs exposed to acute or to chronic hypoxia. Modulation of gene expression by nitric oxide. J. Clin. Invest. 95:1798-1807.

44. McMurtry, A.L., Cho, K., Young, L.J., Nelson, C.F., and Greenhalgh, D.G. 1999. Expression of HSP70 in healing wounds of diabetic and nondiabetic mice. J. Surg. Res. 86:36-41.

45. Liechty, K.W., et al. 1999. Adenoviral-mediated overexpression of platelet-derived growth factor-B corrects ischemic impaired wound healing. J. Invest. Dermatol. 113:375-383. 